\title{
Generic Well-Posedness for a Class of Equilibrium Problems
}

\author{
Alexander J. Zaslavski \\ Department of Mathematics, The Technion-Israel Institute of Technology, 32000 Haifa, Israel
}

Correspondence should be addressed to Alexander J. Zaslavski, ajzasl@tx.technion.ac.il

Received 23 December 2007; Accepted 6 March 2008

Recommended by Simeon Reich

We study a class of equilibrium problems which is identified with a complete metric space of functions. For most elements of this space of functions (in the sense of Baire category), we establish that the corresponding equilibrium problem possesses a unique solution and is well-posed.

Copyright (C) 2008 Alexander J. Zaslavski. This is an open access article distributed under the Creative Commons Attribution License, which permits unrestricted use, distribution, and reproduction in any medium, provided the original work is properly cited.

\section{Introduction}

The study of equilibriumproblems has recently been a rapidly growing area of research. See, for example, [1-3] and the references mentioned therein.

Let $(X, \rho)$ be a complete metric space. In this paper, we consider the following equilibrium problem:

$$
\text { To find } x \in X \text { such that } f(x, y) \geq 0 \quad \forall y \in X \text {, }
$$

where $f$ belongs to a complete metric space of functions $\mathcal{A}$ defined below. In this paper, we show that for most elements of this space of functions $\mathcal{A}$ (in the sense of Baire category) the equilibrium problem $(\mathrm{P})$ possesses a unique solution. In other words, the problem $(\mathrm{P})$ possesses a unique solution for a generic (typical) element of $\mathcal{A}$ [4-6].

Set

$$
\rho_{1}\left(\left(x_{1}, y_{1}\right),\left(x_{2}, y_{2}\right)\right)=\rho\left(x_{1}, x_{2}\right)+\rho\left(y_{1}, y_{2}\right), \quad x_{1}, x_{2}, y_{1}, y_{2} \in X
$$

Clearly, $\left(X \times X, \rho_{1}\right)$ is a complete metric space.

Denote by $\mathcal{A}_{0}$ the set of all continuous functions $f: X \times X \rightarrow R^{1}$ such that

$$
f(x, x)=0 \quad \forall x \in X .
$$


We equip the set $\mathcal{A}_{0}$ with the uniformity determined by the base

$$
U(\epsilon)=\left\{(f, g) \in \mathcal{A}_{0} \times \mathcal{A}_{0}:|f(z)-g(z)| \leq \epsilon \forall z \in X \times X\right\},
$$

where $\epsilon>0$. It is clear that the space $\mathcal{A}_{0}$ with this uniformity is metrizable (by a metric $d$ ) and complete.

Denote by $\mathcal{A}$ the set of all $f \in \mathcal{A}_{0}$ for which the following properties hold.

(P1) For each $\epsilon>0$, there exists $x_{\epsilon} \in X$ such that $f\left(x_{\epsilon}, y\right) \geq-\epsilon$ for all $x \in X$.

(P2) For each $\epsilon>0$, there exists $\delta>0$ such that $|f(x, y)| \leq \epsilon$ for all $x, y \in X$ satisfying $\rho(x, y) \leq \delta$.

Clearly, $\mathcal{A}$ is a closed subset of $X$. We equip the space $A$ with the metric $d$ and consider the topological subspace $\mathscr{A} \subset \mathcal{A}_{0}$ with the relative topology.

For each $x \in X$ and each subset $D \subset X$, put

$$
\rho(x, D)=\inf \{\rho(x, y): y \in D\} \text {. }
$$

For each $x \in X$ and each $r>0$, set

$$
\begin{gathered}
B(x, r)=\{y \in X: \rho(x, y) \leq r\}, \\
B^{o}(x, r)=\{y \in X: \rho(x, y)<r\} .
\end{gathered}
$$

Assume that the following property holds.

(P3) There exists a positive number $\Delta$ such that for each $y \in X$ and each pair of real numbers $t_{1}, t_{2}$ satisfying $0<t_{1}<t_{2}<\Delta$, there is $z \in X$ such that $\rho(z, y) \in\left[t_{1}, t_{2}\right]$.

In this paper, we will establish the following result.

Theorem 1.1. There exists a set $\mathcal{F} \subset \mathcal{A}$ which is a countable intersection of open everywhere dense subsets of $\mathbb{A}$ such that for each $f \in \mathcal{F}$, the following properties hold:

(i) there exists a unique $x_{f} \in X$ such that

$$
f\left(x_{f}, y\right) \geq 0 \quad \forall x, y \in X
$$

(ii) for each $\epsilon>0$, there are $\delta>0$ and a neighborhood $V$ of $f$ in $A$ such that for each $h \in V$ and each $x \in X$ satisfying $\inf \{h(x, y): y \in X\}>-\delta$, the inequality $\rho\left(x_{f}, x\right)<\epsilon$ holds.

In other words, for a generic (typical) $f \in \mathcal{A}$, the problem (P) is well-posed [7-9].

\section{An auxiliary density result}

Lemma 2.1. Let $f \in \mathcal{A}$ and $\epsilon \in(0,1)$. Then there exist $f_{0} \in \mathcal{A}$ and $x_{0} \in X$ such that $\left(f, f_{0}\right) \in U(\epsilon)$ and $f\left(x_{0}, y\right) \geq 0$ for all $y \in X$. 
Proof. By (P1) there is $x_{0} \in X$ such that

$$
f\left(x_{0}, y\right) \geq-\frac{\epsilon}{16} \quad \forall y \in X
$$

Set

$$
\begin{aligned}
& E_{1}=\left\{(x, y) \in X \times X: f(x, y) \geq-\frac{\epsilon}{16}\right\}, \\
& E_{2}=\left\{(x, y) \in(X \times X) \backslash E_{1}: f(x, y) \geq-\frac{\epsilon}{8}\right\}, \\
& E_{3}=(X \times X) \backslash\left(E_{1} \cup E_{2}\right) .
\end{aligned}
$$

For each $\left(y_{1}, y_{2}\right) \in E_{1}$, there is $r_{1}\left(y_{1}, y_{2}\right) \in(0,1)$ such that

$$
f\left(z_{1}, z_{2}\right)>-\frac{\epsilon}{14} \quad \forall z_{1}, z_{2} \in X \text { satisfying } \rho\left(z_{i}, y_{i}\right) \leq r_{1}\left(y_{1}, y_{2}\right), \quad i=1,2 .
$$

For each $\left(y_{1}, y_{2}\right) \in E_{2}$, there is $r_{1}\left(y_{1}, y_{2}\right) \in(0,1)$ such that

$$
f\left(z_{1}, z_{2}\right)>-\frac{\epsilon}{6} \quad \forall z_{1}, z_{2} \in X \text { satisfying } \rho\left(z_{i}, y_{i}\right) \leq r_{1}\left(y_{1}, y_{2}\right), \quad i=1,2 .
$$

For each $\left(y_{1}, y_{2}\right) \in E_{3}$, there is $r_{1}\left(y_{1}, y_{2}\right) \in(0,1)$ such that

$$
f\left(z_{1}, z_{2}\right)<-\frac{\epsilon}{8} \quad \forall z_{1}, z_{2} \in X \text { satisying } \rho\left(z_{i}, y_{i}\right) \leq r_{1}\left(y_{1}, y_{2}\right), \quad i=1,2 .
$$

For each $\left(y_{1}, y_{2}\right) \in X \times X$, set

$$
U\left(y_{1}, y_{2}\right)=B^{o}\left(y_{1}, r_{1}\left(y_{1}, y_{2}\right)\right) \times B^{o}\left(y_{2}, r_{1}\left(y_{1}, y_{2}\right)\right)
$$

For any $\left(y_{1}, y_{2}\right) \in E_{1} \cup E_{2}$, put

$$
g_{y_{1}, y_{2}}(z)=\max \{f(z), 0\}, \quad z \in X \times X
$$

and for any $\left(y_{1}, y_{2}\right) \in E_{3}$, put

$$
g_{y_{1}, y_{2}}(z)=f(z), \quad z \in X \times X
$$

Clearly, $\left\{U\left(y_{1}, y_{2}\right): y_{1}, y_{2} \in X\right\}$ is an open covering of $X \times X$. Since any metric space is paracompact, there is a continuous locally finite partition of unity $\left\{\phi_{\beta}: \beta \in \mathbb{B}\right\}$ subordinated to the covering $\left\{U\left(y_{1}, y_{2}\right): y_{1}, y_{2} \in X\right\}$. Namely, for any $\beta \in \mathbb{B}, \phi_{\beta}: X \times X \rightarrow[0,1]$ is a continuous function and there exist $y_{1}(\beta), y_{2}(\beta) \in X$ such that $\operatorname{supp}\left(\phi_{\beta}\right) \subset U\left(y_{1}(\beta), y_{2}(\beta)\right)$ and that

$$
\sum_{\beta \in B} \phi_{\beta}(z)=1 \quad \forall z \in X \times X
$$

Define

$$
f_{0}(z)=\sum_{\beta \in \mathcal{B}} \phi_{\beta}(z) g_{\left(y_{1}(\beta), y_{2}(\beta)\right)}(z), \quad z \in X \times X .
$$


Clearly, $f_{0}$ is well defined, continuous, and satisfies

$$
f_{0}(z) \geq f(z) \quad \forall z \in X \times X
$$

Let $\left(z_{1}, z_{2}\right) \in E_{1}$. Then

$$
f\left(z_{1}, z_{2}\right) \geq-\frac{\epsilon}{16}
$$

Assume that $\beta \in \mathbb{B}$ and that $\phi_{\beta}\left(z_{1}, z_{2}\right)>0$. Then

$$
\left(z_{1}, z_{2}\right) \in \operatorname{supp}\left(\phi_{\beta}\right) \subset U\left(y_{1}(\beta), y_{2}(\beta)\right)
$$

If $\left(y_{1}(\beta), y_{2}(\beta)\right) \in E_{3}$, then in view of (2.5), (2.6), and (2.13), $f\left(z_{1}, z_{2}\right)<-\epsilon / 8$, a contradiction (see (2.12)). Then $\left(y_{1}(\beta), y_{2}(\beta)\right) \in E_{1} \cup E_{2}$, and by (2.7),

$$
g_{y_{1}(\beta), y_{2}(\beta)}\left(z_{1}, z_{2}\right)=\max \left\{f\left(z_{1}, z_{2}\right), 0\right\} .
$$

Since this equality holds for any $\beta \in \mathbb{B}$ satisfying $\phi_{\beta}\left(z_{1}, z_{2}\right)>0$, it follows from (2.10) that

$$
f_{0}\left(z_{1}, z_{2}\right)=\max \left\{f\left(z_{1}, z_{2}\right), 0\right\}
$$

for all $\left(z_{1}, z_{2}\right) \in E_{1}$.

Relations (2.1), (2.2), and (2.15) imply that

$$
f_{0}\left(x_{0}, y\right) \geq 0, \quad y \in X
$$

By (1.2), (2.7), (2.8), and (2.10)

$$
f_{0}(x, x)=0, \quad x \in X
$$

Assume that

$$
\left(z_{1}, z_{2}\right) \in E_{2}
$$

Then in view of (2.2) and (2.18), $f\left(z_{1}, z_{2}\right) \geq-\epsilon / 8$. Together with (2.7) and (2.10), this implies that

$$
f_{0}\left(z_{1}, z_{2}\right) \leq \sum_{\beta \in \mathcal{B}} \phi_{\beta}\left(z_{1}, z_{2}\right)\left(f\left(z_{1}, z_{2}\right)+\frac{\epsilon}{8}\right)=f\left(z_{1}, z_{2}\right)+\frac{\epsilon}{8}
$$

Combined with (2.11), this implies that

$$
f\left(z_{1}, z_{2}\right) \leq f_{0}\left(z_{1}, z_{2}\right) \leq f\left(z_{1}, z_{2}\right)+\frac{\epsilon}{8}
$$

for all $\left(z_{1}, z_{2}\right) \in E_{2}$. 
Let

$$
\left(z_{1}, z_{2}\right) \in E_{3}
$$

and assume that

$$
\beta \in \mathbb{B}, \quad \phi_{\beta}\left(z_{1}, z_{2}\right)>0
$$

Then in view of (2.22),

$$
\left(z_{1}, z_{2}\right) \in \operatorname{supp}\left(\phi_{\beta}\right) \subset U\left(y_{1}(\beta), y_{2}(\beta)\right)
$$

By (2.23) and the choice of $U\left(y_{1}(\beta), y_{2}(\beta)\right)$ (see (2.3)-(2.6)), $\left(y_{1}(\beta), y_{2}(\beta)\right) \notin E_{1}$ and by (2.4), (2.6), (2.7), and (2.8),

$$
g_{y_{1}(\beta), y_{2}(\beta)}\left(z_{1}, z_{2}\right) \leq f\left(z_{1}, z_{2}\right)+\frac{\epsilon}{6} .
$$

Since the inequality above holds for any $\beta \in \mathbb{B}$ satisfying (2.22), the relation (2.10) implies that

$$
f_{0}\left(z_{1}, z_{2}\right) \leq f\left(z_{1}, z_{2}\right)+\frac{\epsilon}{6}
$$

Together with (2.11), (2.12), and (2.15), this implies that for all $\left(z_{1}, z_{2}\right) \in X \times X$

$$
f\left(z_{1}, z_{2}\right) \leq f_{0}\left(z_{1}, z_{2}\right) \leq f\left(z_{1}, z_{2}\right)+\frac{\epsilon}{6} .
$$

By (2.17), $f_{0} \in \mathcal{A}_{0}$. In view of (2.16), $f_{0}$ possesses (P1). Since $f$ possesses (P2), it follows from (2.7), (2.8), and (2.10) that $f_{0}$ possesses (P2). Therefore $f_{0} \in \mathcal{A}$ and Lemma 2.1 now follows from (2.16) and (2.26).

\section{A perturbation lemma}

Lemma 3.1. Let $\epsilon \in(0,1), f \in \mathcal{A}$, and let $x_{0} \in X$ satisfy

$$
f\left(x_{0}, y\right) \geq 0 \quad \forall y \in X
$$

Then there exist $g \in \mathcal{A}$ and $\delta>0$ such that

$$
g\left(x_{0}, y\right) \geq 0 \quad \forall y \in X, \quad|(g-f)(x, y)| \leq \frac{\epsilon}{4} \quad \forall x, y \in X
$$

and if $x \in X$ satisfies $\inf \{g(x, y): y \in X\}>-\delta$, then $\rho\left(x_{0}, x\right)<\epsilon / 8$.

Proof. By (P2) there is a positive number

$$
\delta_{0}<\min \left\{16^{-1} \epsilon, 16^{-1} \Delta\right\}
$$

such that

$$
|f(y, z)| \leq \frac{\epsilon}{16} \quad \forall y, z \in X \text { satisfying } \rho(y, z) \leq 4 \delta_{0}
$$


Set

$$
\delta=2^{-1} \delta_{0}
$$

Define

$$
\begin{gathered}
\phi(t)=1, \quad t \in\left[0, \delta_{0}\right], \\
\phi(t)=0, \quad t \in\left[2 \delta_{0}, \infty\right), \\
\phi(t)=2-t \delta_{0}^{-1}, \quad t \in\left(\delta_{0}, 2 \delta_{0}\right), \\
f_{1}(x, y)=-\phi(\rho(x, y)) \rho(x, y)+(1-\phi(\rho(x, y))) f(x, y), \quad(x, y \in X) .
\end{gathered}
$$

Clearly, $f_{1}$ is continuous and

$$
f_{1}(x, x)=0 \quad \forall x \in X
$$

By (3.6) and (3.7),

$$
f_{1}(x, y)=-\rho(x, y) \quad \forall x, y \in X \text { satisfying } \rho(x, y) \leq \delta_{0} .
$$

Let $x, y \in X$. We estimate $\left|f(x, y)-f_{1}(x, y)\right|$. If $\rho(x, y) \geq 2 \delta_{0}$, then by (3.6) and (3.7),

$$
\left|f_{1}(x, y)-f(x, y)\right|=0
$$

Assume that

$$
\rho(x, y) \leq 2 \delta_{0}
$$

By (3.3) and (3.11),

$$
|f(x, y)| \leq \frac{\epsilon}{16} .
$$

By (3.5), (3.6), (3.7), (3.11), and (3.12),

$$
\left|f_{1}(x, y)-f(x, y)\right| \leq \rho(x, y)+|f(x, y)| \leq 2 \delta_{0}+\frac{\epsilon}{16}<\frac{\epsilon}{4} .
$$

Together with (3.10) this implies that

$$
\left|f_{1}(x, y)-f(x, y)\right|<\frac{\epsilon}{4} \quad \forall x, y \in X
$$

Assume that $x \in X$. In view of (P3) and (3.3), there is $y \in X$ such that

$$
\rho(y, x) \in\left[2^{-1} \delta_{0}, \delta_{0}\right]
$$

It follows from (3.15) and (3.9) that

$$
\begin{array}{r}
f_{1}(x, y)=-\rho(y, x) \leq-2^{-1} \delta_{0} \\
\inf \left\{f_{1}(x, z): z \in X\right\} \leq-2^{-1} \delta_{0}
\end{array}
$$


for all $x \in X$. Set

$$
g(x, y)=\phi\left(\rho\left(x, x_{0}\right)\right) f(x, y)+\left(1-\phi\left(\rho\left(x, x_{0}\right)\right)\right) f_{1}(x, y), \quad x, y \in X .
$$

Clearly, the function $g$ is continuous and

$$
g(x, x)=0 \quad \forall x \in X
$$

In view of (3.1), (3.18), and (3.6),

$$
g\left(x_{0}, y\right)=f\left(x_{0}, y\right) \geq 0 \quad \forall y \in X
$$

Since the function $f$ possesses (P2), it follows from (3.9), (3.20), and (3.18) that $g$ possesses the property (P2). Thus $g \in \mathcal{A}$.

By (3.6), (3.14), and (3.18) for all $x, y \in X$

$$
|(f-g)(x, y)| \leq\left|f_{1}(x, y)-f(x, y)\right| \leq \frac{\epsilon}{4} .
$$

Assume that

$$
x \in X, \quad \inf \{g(x, y): y \in X\}>-2^{-1} \delta_{0}=-\delta .
$$

If $\rho\left(x_{0}, x\right) \geq 2 \delta_{0}$, then by (3.6) and (3.18),

$$
g(x, y)=f_{1}(x, y) \quad \forall y \in Y
$$

and together with (3.17), this implies that

$$
\inf \{g(x, y): y \in X\} \leq-2^{-1} \delta_{0} .
$$

This inequality contradicts (3.22). The contradiction we have reached proves that

$$
\rho\left(x_{0}, x\right)<2 \delta_{0}<\frac{\epsilon}{8} .
$$

This completes the proof of the lemma.

\section{Proof of Theorem 1.1}

Denote by $E$ the set of all $f \in \mathcal{A}$ for which there exists $x \in X$ such that $f(x, y) \geq 0$ for all $y \in X$. By Lemma 2.1, $E$ is an everywhere dense subset of $\mathcal{A}$.

Let $f \in E$ and $n$ be a natural number. There exists $x_{f} \in X$ such that

$$
f\left(x_{f}, y\right) \geq 0 \quad \forall y \in X
$$

By Lemma 3.1, there exist $g_{f, n} \in \mathcal{A}$ and $\delta_{f, n}>0$ such that

$$
g_{f, n}\left(x_{f}, y\right) \geq 0 \quad \forall y \in X, \quad\left|\left(g_{f, n}-f\right)(x, y)\right| \leq(4 n)^{-1} \quad \forall x, y \in X,
$$

and the following property holds. 
(P4) For each $x \in X$ satisfying $\inf \left\{g_{f, n}(x, y): y \in X\right\}>-\delta_{f, n}$, the inequality $\rho\left(x_{f}, x\right)<$ $(4 n)^{-1}$ holds.

Denote by $V(f, n)$ the open neighborhood of $g_{f, n}$ in $\mathcal{A}$ such that

$$
V(f, n) \subset\left\{h \in \mathcal{A}:\left(h, g_{f, n}\right) \in U\left(4^{-1} \delta_{f, n}\right)\right\}
$$

Assume that

$$
x \in X, \quad h \in V(f, n), \quad \inf \{h(x, y): y \in X\}>-2^{-1} \delta_{f, n} .
$$

By (1.3), (4.3), and (4.4),

$$
\inf \left\{g_{f, n}(x, y): y \in X\right\} \geq \inf \{h(x, y): y \in X\}-4^{-1} \delta_{f, n}>-\delta_{f, n}
$$

In view of (4.5) and (P4),

$$
\rho\left(x_{f}, x\right)<(4 n)^{-1}
$$

Thus we have shown that the following property holds. holds.

(P5) For each $x \in X$ and each $h \in V(f, n)$ satisfying $(4.4)$, the inequality $\rho\left(x_{f}, x\right)<(4 n)^{-1}$ Set

$$
\mathcal{F}=\bigcap_{k=1}^{\infty} \cup\{V(f, n): f \in E \text { and an integer } n \geq k\} .
$$

Clearly, $\mathcal{F}$ is a countable intersection of open everywhere dense subset of $\mathcal{A}$. Let

$$
\xi \in \mathcal{F}, \quad \epsilon>0 .
$$

Choose a natural number $k>8\left(\epsilon^{-1}+1\right)$. There exist $f \in E$ and an integer $n \geq k$ such that

$$
\xi \in V(f, n)
$$

The property (P4), (4.3), and (4.9) imply that for each $x \in X$ satisfying

$$
\inf \{\xi(x, y): y \in X\}>-2^{-1} \delta_{f, n}
$$

we have

$$
\begin{gathered}
\inf \left\{g_{f, n}(x, y): y \in X\right\}>-2^{-1} \delta_{f, n}-4^{-1} \delta_{f, n}>-\delta_{f, n}, \\
\rho\left(x_{f}, x\right)<(4 n)^{-1}<\frac{\epsilon}{8} .
\end{gathered}
$$

Thus we have shown that the following property holds.

(P6) For each $x \in X$ satisfying (4.10), the inequality $\rho\left(x_{f}, x\right)<\epsilon / 8$ holds. 
By (P1) there is a sequence $\left\{x_{i}\right\}_{i=1}^{\infty} \subset X$ such that

$$
\liminf _{i \rightarrow \infty}\left(\inf \left\{\xi\left(x_{i}, y\right): y \in X\right\}\right) \geq 0 .
$$

In view of (4.12) and (P6) for all large enough natural numbers $i, j$, we have

$$
\rho\left(x_{i}, x_{j}\right) \leq \rho\left(x_{i}, x_{f}\right)+\rho\left(x_{f}, x_{j}\right)<\frac{\epsilon}{4} .
$$

Since $\epsilon$ is any positive number, we conclude that $\left\{x_{i}\right\}_{i=1}^{\infty}$ is a Cauchy sequence and there exists

$$
x_{\xi}=\lim _{i \rightarrow \infty} x_{i}
$$

Relations (4.12) and (4.14) imply that for all $y \in X$

$$
\xi\left(x_{\xi}, y\right)=\lim _{i \rightarrow \infty} \xi\left(x_{i}, y\right) \geq 0 .
$$

We have also shown that any sequence $\left\{x_{i}\right\}_{i=1}^{\infty} \subset X$ satisfying (4.12) converges. This implies that if $x \in X$ satisfies $\xi(x, y) \geq 0$ for all $y \in X$, then $x=x_{\xi}$. By (P6) and (4.15),

$$
\rho\left(x_{\xi}, x_{f}\right) \leq \frac{\epsilon}{8} .
$$

Let $x \in X$ and $h \in V(f, n)$ satisfy (4.4). By (P5), $\rho\left(x_{f}, x\right)<(4 n)^{-1}$. Together with (4.16), this implies that

$$
\rho\left(x, x_{\xi}\right) \leq \rho\left(x, x_{f}\right)+\rho\left(x_{f}, x_{\xi}\right)<(4 n)^{-1}+\frac{\epsilon}{8}<\epsilon .
$$

Theorem 1.1 is proved.

\section{References}

[1] E. Blum and W. Oettli, "From optimization and variational inequalities to equilibrium problems," The Mathematics Student, vol. 63, no. 1-4, pp. 123-145, 1994.

[2] G.-Y. Chen, X. Huang, and X. Yang, Vector Optimization, Set-Valued and Variational Analysis, Springer, Berlin, Germany, 2005.

[3] X. J. Long, N.-J. Huang, and K. L. Teo, "Levitin-Polyak well-posedness for equilibrium problems with functional constraints," Journal of Inequalities and Applications, vol. 2008, Article ID 657329, 14 pages, 2008.

[4] J.-P. Aubin and I. Ekeland, Applied Nonlinear Analysis, John Wiley \& Sons, New York, NY, USA, 1984.

[5] Z. Nitecki, Differentiable Dynamics. An Introduction to the Orbit Structure of Diffeomorphisms, MIT Press, Cambridge, Mass, USA, 1971.

[6] A. J. Zaslavski, Turnpike Properties in the Calculus of Variations and Optimal Control, Springer, New York, NY, USA, 2006.

[7] A. D. Ioffe and A. J. Zaslavski, "Variational principles and well-posedness in optimization and calculus of variations," SIAM Journal on Control and Optimization, vol. 38, no. 2, pp. 566-581, 2000.

[8] A. J. Zaslavski, "Generic well-posedness of optimal control problems without convexity assumptions," SIAM Journal on Control and Optimization, vol. 39, no. 1, pp. 250-280, 2000.

[9] T. Zolezzi, "Well-posedness criteria in optimization with application to the calculus of variations," Nonlinear Analysis: Theory, Methods \& Applications, vol. 25, no. 5, pp. 437-453, 1995. 